\title{
FORGE Toolkit: Leveraging Distributed Systems in eLearning Platforms
}

\author{
GUILLAUME JOURJON ${ }^{1}$, JOHANN M. MARQUEZ-BARJA ${ }^{2}$, THIERRY RAKOTOARIVELO', \\ ALEXANDER MIKROYANNIDIS ${ }^{3}$, KOSTAS LAMPROPOULOS ${ }^{4}$, SPYROS DENAZIS ${ }^{4}$, \\ CHRISTOS TRANORIS ${ }^{4}$, DAAN PAREIT ${ }^{5}$, JOHN DOMINGUE ${ }^{3}$, \\ LUIZ A. DASILVA ${ }^{2,6}$, AND MAX OTT ${ }^{1}$ \\ ${ }^{1}$ Data61-Commonwealth Scientific and Industrial Research Organisation, Sydney, NSW 2350, Australia \\ ${ }^{2}$ CONNECT-Trinity College Dublin, Dublin 2, Ireland \\ ${ }^{3}$ The Open University, Buckinghamshire MK7 6AA, U.K. \\ ${ }^{4}$ University of Patras, Patras 265 04, Greece

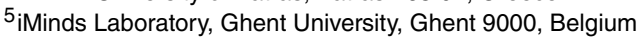 \\ ${ }^{6}$ Virginia Tech, Blacksburg, VA 24061 USA \\ CORRESPONDING AUTHOR: G. JOURJON (guillaume.jourjon@ nicta.com.au)
}

This work was supported in part by the European Union's Seventh Framework Programme for Research, Technological Development and Demonstration under Grant 610889 and in part by the Science Foundation Ireland under Grant 10/CE/l1853.

\begin{abstract}
While more and more services become virtualized and always accessible in our society, laboratories supporting computer science (CS) lectures have mainly remained offline and class-based. This apparent abnormality is due to several limiting factors, discussed in the literature, such as the high cost of deploying and maintaining computer network testbeds and the lack of standardization for the presentation of eLearning platforms. In this paper, we present the FORGE toolkit, which leverages experimentation facilities currently deployed in international initiatives for the development of e-learning materials. Thus, we solve the institutional challenge mentioned in the ACM/IEEE 2013 CS curricula concerning the access and maintenance of specialized and heterogeneous hardware thanks to a seamless integration with the networking test-bed community. Moreover, this project builds an ecosystem, where teaching and educational materials, tools, and experiments are available under open scheme and policies. We demonstrate how it already meets most of the requirements from the network and communication component of CS 2013 and some of the labs of the Cisco academy. Finally, we present experience reports illustrating the potential benefits of this framework based on the first deployments in four post-graduate courses in prestigious institutions around the world.
\end{abstract}

INDEX TERMS eLearning, networking, testbeds.

\section{INTRODUCTION}

In 2013, after more than 10 years, the ACM, in collaboration with the IEEE, presented an updated version of the Computer Science curricula [1]. This new version paves the way for educating the next generation of computer scientists while also identifying critical challenges for institutions and students. In particular, the 2013 version recognises the ubiquitous role of Networks and new technologies in 'today's and tomorrow's computing environment." This also brings some severe institutional challenges for providing the rich environments as described in Chapter 6 of the curricula.

Programs in computer science have a need for adequate computing resources, both for students and faculty. The needs of computer science programs often extend beyond traditional infrastructure (general campus computing labs) and may include specialized hardware and software, and/or large-scale computing infrastructure. Having adequate access to such resources is especially important for project and capstone courses. Moreover, institutions need to consider the growing heterogeneity of computing devices (e.g., smartphones, tablets) that can be used as a platform for coursework.

ACM/IEEE CS Curricula 2013, p. 51.

A similar resource problem has been tackled by the network and distributed systems research communities over the last fifteen years. The need for large scale infrastructure 
to perform equally large experiments resulted in various initiatives around the world. The most prominent have been Global Environment for Network Innovations (GENI) [2] and Future Internet Research and Experimentation (FIRE) [3] initiatives in the US and Europe, respectively. These projects first focused on the deployment and management of large federated networking testbeds for the development and experimentation of Future Internet technologies, also allowing non-networking academics and researchers to use these facilities. Indeed, going beyond what these projects originally intended to demonstrate, researchers from various fields have also used these resources for testing their algorithms and architectures such as the Collaborative Adaptive Sensing of the Atmosphere (CASA) project. ${ }^{1}$

A natural use of resources provided by these federations was to leverage the near ubiquitous availability of heterogeneous hardware to try to solve the computing resource problem mentioned above. Therefore, several projects embarked on the definition of a new learning layer on top of these distributed resources specially targeted at educating future engineers [4]-[9]. While these projects succeeded at deploying networking labs and exploiting the underlying network offered by the testbed community, they failed to reach a critical number of users in the learning community. Thus, even if it has been demonstrated in [7] and [9] that this approach could be beneficial to students to better understand global and specific concepts of a lecture, these approaches do not often spread beyond their original learning institutions. One of the hurdles for broader use of these systems is the difficulty for teachers to install these tools and develop new content for their specific needs. Furthermore, deploying new standalone tools requires an additional learning step for students to navigate through an unfamiliar interface [7], [9].

In this paper, we present the FORGE toolkit, which aims to leverage the experimentation facilities from the FIRE and GENI programs for the development of e-learning materials as depicted in Fig. 1. Moreover, this project builds an ecosystem where teaching and educational materials,

\footnotetext{
${ }^{1}$ http://www.casa.umass.edu/
}

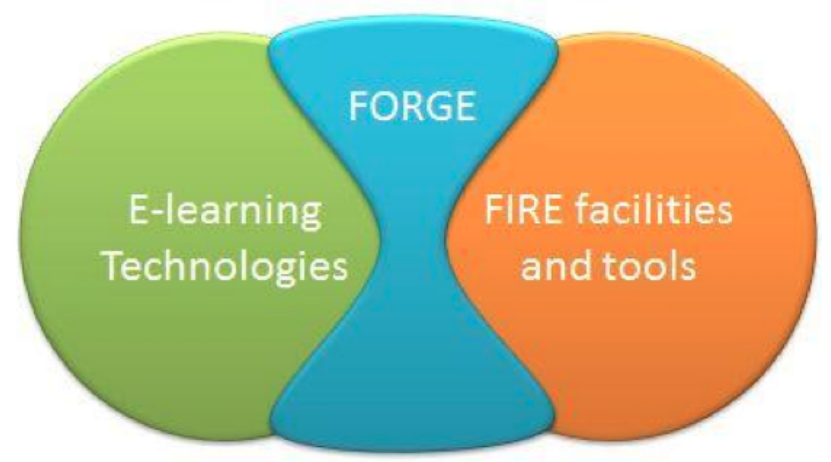

FIGURE 1. Interfacing research and learning communities with the FORGE project. tools and experiments are available under an open scheme and policies. This paper demonstrates the many advantages of this framework compared to existing solutions. In particular we demonstrate:

1) how our proposal leverages existing tools and user interfaces while seamlessly integrating resources from a large pool of federated facilities, and therefore is not yet another standalone eLearning tool;

2) how it already covers most of the requirements from the Network and Communication component of CS 2013 and some of the labs of the Cisco academy;

3) the benefits of our framework based on the experience reports from four post-graduate courses in universities around the world.

The remainder of the paper is organised as follows. Section II presents related work on large scale testbed facilities and how they have been used to provide interactive labs. In Section III, we detail how we use well-known e-learning tools such as e-books and Learning Management System (LMS) to interact with these infrastructures. We also detail how we match both the ACM Curriculum and the Cisco academy requisites. We describe first deployments of these labs in Section IV. Finally, Section V presents our concluding remarks.

\section{RELATED WORK}

\section{A. EDUCATIONAL APPROACHES}

A wide range of studies have shown that with the right scaffolding competent learners benefit greatly from constructivist or learning-by-doing approaches [10]-[13]. In another currently ongoing FP7 project, Working Environment with Social, Personal and Open Technologies for Inquiry Based Learning (weSPOT), partners focus on Inquiry Based Learning (IBL), by propagating scientific inquiry as the approach for science learning and teaching in combination with today's curricula and teaching practices.

The notion of Self-Regulated Learning (SRL) can also be promoted through the use and propagation of FIRE learning solutions in online education. SRL has become increasingly important in educational and psychological research. The goal is to give the learner greater responsibility and control over all aspects of technology-enhanced learning. Lately, as we have learned from our involvement in the FP7 projects Responsive Open Learning Environments (ROLE) and Sustaining Technology Enhanced Learning at a LARge scale (STELLAR), the advance of lifelong and informal learning has made SRL important in non-academic learning environments, where instead of instructor- and teacher-orientation, more learner-orientation is necessary [14].

The second trend that we see is based on Linked Data [15], [16], the simplest form of the Semantic Web [17], where data representing any entity (e.g. person, place or organization) is identified by a Uniform Resource Identifier (URI) and can be linked to any other data item, forming a gigantic Web of Data graph. In general Linked Data is being used by companies such as Google, Microsoft and 
Yahoo to enhance web search, ${ }^{2}$ by Facebook to support the linking of social media resources ${ }^{3}$ and by the BBC to support the delivery of online news content. ${ }^{4}$

Regarding the learning assessment methods, there are different tools for qualitative and quantitative evaluation, such as surveys and learning analytics, respectively [18]. Diwakar et al. [19] analized the relation among several components between technology and pedagogy; considering testbed technology, processing capabilities, and user interfaces, and feedback methods, within the control systems domain. Authors took into account several works regarding remote experimentation laboratories, including a wide range of technologies such as Matlab systems or web-based interfaces.

\section{B. CONTROLLING EXPERIMENTS IN THE WILD}

Running experiments over large testbeds is a challenging task, as it involves the provisioning, orchestration and instrumentation of many heterogeneous distributed resources. Some recent contributions have proposed concise protocols, information models, and interfaces to remotely control and collect measurements from such resources, such as the Federated Resource Control Protocol (FRCP) and the OML Measurement Stream Protocol (OMSP) [20], [21].

FRCP [20] defines the interaction between experiment controller and resource controller entities using a small set of REST-like messages. These interactions are asynchronous and use a publish and subscribe system for added robustness. These two aspects provide support for long term or disconnected experiments (i.e. any controllers might be offline for any duration) and large scale ones (i.e. thousands of distributed resources), respectively. FRCP has been implemented in both the cOntrol and Management Framework (OMF) [22] and the NEPI experimenter tools [23], which have been used to run many experiments on various GENI and FIRE testbeds.

OMSP [21] describes the format and transport of measurement tuples between injection points (e.g. an application resource) and collection points (e.g. a storage server). It supports multiple data formats (e.g. strings, numerics, binary blobs, vectors), two types of encoding (binary and text), and metadata injection. OMSP is implemented in the Orbit Measurement Library (OML) software suite, which provides a collection point server and a client-side library in $\mathrm{C}$ to instrument resources. In addition, several third parties have provided client-side libraries for other languages such as Python or Java, and have instrumented many applications.

\section{TESTBED FEDERATION AND AUTHENTICATION}

The GENI Federation Architecture [2] consists of a set of rules describing the rights and obligations of testbeds as well

\footnotetext{
${ }^{2} \mathrm{http} / / / \mathrm{schema.org}$

${ }^{3}$ http://ogp.em/

${ }^{4}$ http://www.bbc.co.uk/blogs/bbcinternet/2010/07/bbc_world_cup_2010 _dynamic_sem.html
}

as users joining the federation. In addition, it defines a set of services to facilitate the interaction between the testbeds and the users according to those rules.

The services themselves can be split into those provided by each testbed (also referred to as 'aggregate') directly and those provided by the federation itself. The latter are collectively referred to as the ClearingHouse $(\mathrm{CH})$. These services are primarily concerned with user management and the fair allocation of resources among them. Both are essentially 'codified' expressions of federation rules.

Unfortunately, there are problems with the current design implementation in the context described in this paper. While user authentication can be delegated to identity providers such as InCommon, users of GENI resources have to be added to GENI projects and therefore individually managed within an $\mathrm{CH}$ service. Linking membership management to an educational organisation's 'enrolment' service, such as Internet2's Grouper ${ }^{5}$ would simplify student management.

The more serious problem is that none of the testbed federations we are aware of currently offers any resource allocation guarantees necessary for running a lab with firm submission deadlines. Currently educators are relying on ad-hoc arrangements with individual testbeds or ingenious uses of offered services. Fortunately, these are primarily policy and non-technical issues and we believe that the technical foundations can accommodate the use case proposed in this paper.

\section{TESTBEDS AND ELEARNING}

Past projects have shown, as discussed earlier, how the infrastructure problem mentioned in the CS curricula [1] could be solved. Thus, several projects have already proposed some innovative platforms based on research projects. For instance, "netkit" [24] and its companion project "auto-netkit" [25] use emulated networks and virtual machines to create large topologies and study their control plane at large scales. With a more 'real world' angle in mind, the Seattle project [5] offers educational access to PlanetLab [26] through a browserembedded terminal plugin in order to deploy and test students' assignments.

In [8], the authors have proposed Internet Remote Emulation Experiment Laboratory (IREEL), a web-based interface to deploy predefined experiments on top of federated testbeds while allowing the curious student to see what is under the hood. They have further demonstrated in [7] the effectiveness of this approach based on evaluation from the students. Overall the students rated this project well for understanding general and specific subjects from the lecture. Nevertheless, their approach suffered from a kind of "cold start" effect as the student appeared to be confused by a new interface to perform labs.

The GENI Education project [4] offers a guide to use GENI's resources in a series of labs similar to the ones offered

\footnotetext{
${ }^{5} \mathrm{http}: / /$ www.internet2.edu/products-services/trust-identity-middleware/ grouper/
} 
in [7]. Nevertheless, this project requires both the student and the instructor to be familiar with GENI's provisioning and controlling tools. Therefore, it might not be adequate for all students and teachers.

Recently, testbed facilities have been used to teach other aspects of the ACM Curricula. In [9], the authors have demonstrated how to use GENI's resources, and in particular the GENICloud project, to teach Cloud Computing and Distributed Systems. They found that, while students appreciated the overall approach, some were frustrated by the user interface.

Overall, these approaches suffer from a lack of standardisation in terms of User Interfaces while we are witnessing a protocol consolidation for the provisioning and control of resources on both the GENI and FIRE federations of testbeds. This may lead to sub-optimal benefits for both the educator and the student as they will have to re-learn how to perform experiments for each of these tools. Our proposal aims at solving this issue by not developing yet another new standalone tool but focusing on interfacing well-known teaching tools, such as e-Books and LMSs, to this unified federation of testbeds.

\section{FRAMEWORK ARCHITECTURE}

Recently, several works have demonstrated the potential benefit of using e-books for students [27] as well as the ease to develop new interactive content for CS instructors [28]. Nevertheless, the creation of interactive elements within these platforms requires considerable programming skills as well as familiarity with various authentication and authorisation schemes. In this section, we advance this approach further by demonstrating how to integrate widgets, which are microapplications performing a dedicated task, to control GENI and FIRE resources within an e-book. We then briefly show how we integrated Authentication and Authorisation mechanisms in a transparent manner. We also describe our evaluation process. Finally, we present the currently available widgets matching both the ACM CS Curriculum and the Cisco Academy course.

\section{A. INTEGRATION WITH e-BOOKS AND LMS}

We have combined tools adopted by the FIRE and GENI experimentation communities with those developed by the eLearning community. While testbed Application Programming Interface (API) and tools already provide a way to interact with different testbed facilities, as detailed in Section II, there is a need to develop a number of service wrappers, called testbed adapters, to be able to address these APIs and tools appropriately from within (mostly web based) educational platforms. When developing these adapters some of the most noticeable challenges are:

i making the reservation of resources in (different) facilities easy for both teachers and learners;

ii allowing fast experimentation control;

iii allowing multiple users to share the same experiment; iv knowing the identity of the user who is currently performing an experiment that is initiated from within a client web browser;

$\mathrm{v}$ accessing resources that can only be reached over IPv6 or over a VPN;

vi avoiding breaking the logical flow of an educational experiment when the user behaves unexpectedly;

vii handling a large number of simultaneous users.

From the eLearning perspective, another important aspect is the integration with e-books. In particular, the Apple iBooks is an e-book format which allows the embedding of interactive elements, such as HTML5 widgets, inside an e-book. This enables it to feature dynamic content offered by external online sources. Thus educators can leverage this functionality to offer a richer version of their lectures as demonstrated in [28]. While other e-book formats such as ePub3 also have promising interactive features, they are not mature nor are they fully supported in e-readers yet.

The authoring software that is used for this purpose is the iBooks Author, an application for MacOS available to download for free [29]. The application contains templates for a large number of interactive containers including galleries, quizzes, keynote presentations, and interactive images. Alternatively, users can create and embed their own HTML5 widgets as explained in [28]. In the context of our project, we make full use of the interactive capabilities offered by the iBooks format, by developing and embedding in our e-books interactive self-assessment exercises, such as quizzes, as well as HTML5 widgets that offer access to FIRE facilities in a transparent manner for the students. Furthermore, based on the iBook widget, the LMS integration is straightforward as widgets are web-ready, using exclusively technologies like HTML5 and JavaScript. Fig. 2 shows the

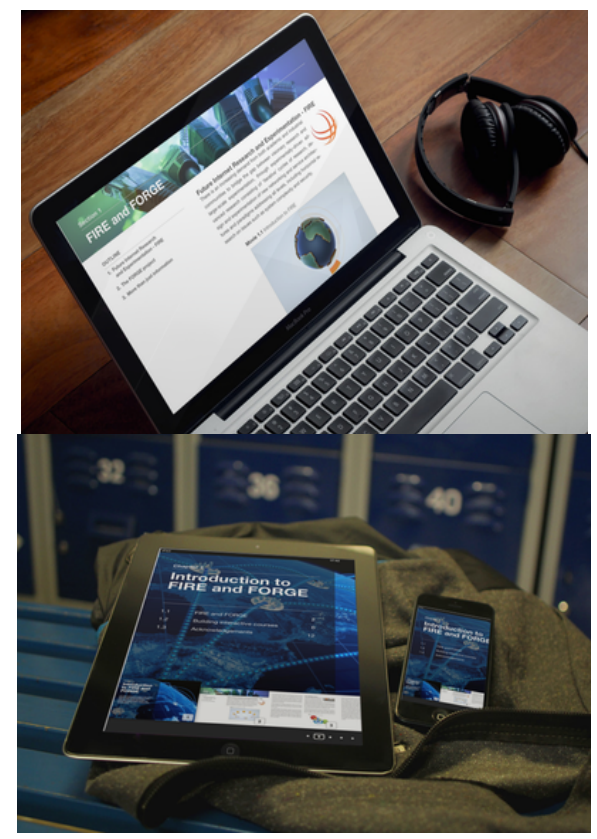

FIGURE 2. FORGE iBook displayed in different types of devices. 


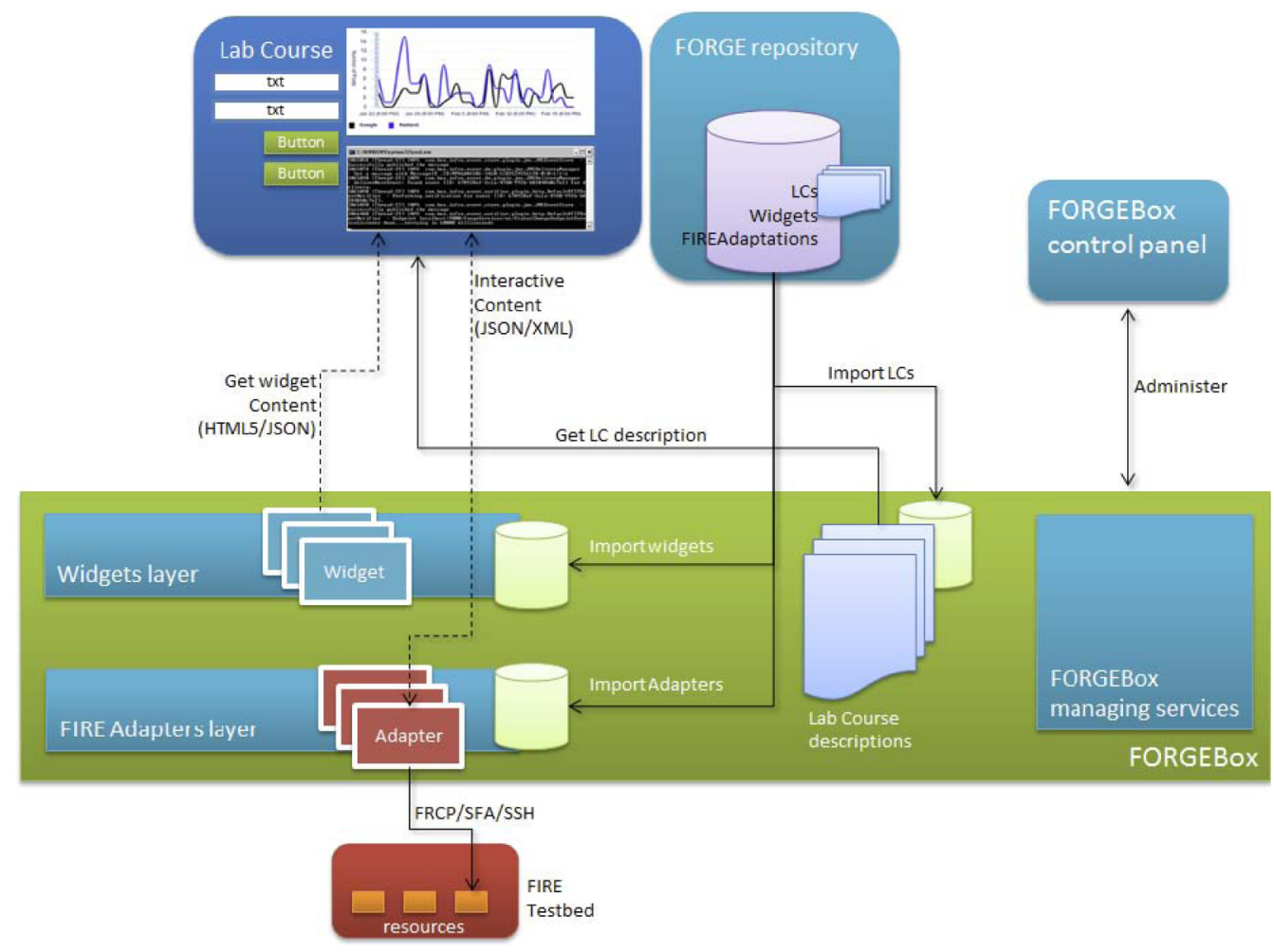

FIGURE 3. Interface facilities and learners.

FORGE eBook deployed and being used in different types of devices.

We present in Fig. 3 our interfacing technology in its birds-eye architectural view, interconnecting learning interactive content with testbed resources. It comprises a set of services that provide and host the interactive content of widgets, and interface them with the physical testbed resources via the adapters. LMSs, e-books and any future element that wishes to consume our content, will need to discover anchor points of widgets and lab course descriptions. We allow the creation of instantiations, called FORGEBoxes, which can host such interactive content.

Fig. 3 provides a detailed view of the FORGEBox architecture. The figure also displays the concept of a FORGE repository that will host any shared published items such as Lab Courses, widgets and FIRE adapters to be used by the learning community and by other organizations hosting a FORGEBox instantiation. At its simplest form, the core consists of services that make some tasks easier such as creating, managing and operating Lab Courses and their content as well as widgets and adapters. FORGEBox contains a set of managing services, a Widgets layer, a FIRE adapters layer and a local repository of hosted Lab Courses. Finally, FORGEBox is implemented under Open Source licenses.

As Fig. 3 presents, a widget is usually hosted inside a web container. This web container might be a regular web server like Apache or even a web service like Tomcat.
Widgets can be reused in an LMS environment or iBook content. Thus, widgets are primarily implemented as HTML/ JavaScript bundles. Widgets are automatically downloaded on the students's device and can then request and retrieve content via specific testbed adapters in a remote FORGEBox instantiation. As Fig. 3 shows, the primary interaction protocol between widgets and adapters is envisioned to be simple Hypertext Transfer Protocol (HTTP) requests/responses with Extensible Markup Language (XML) or JavaScript Object Notation (JSON) payload. As adapters are being addressed via these HTTP requests, they typically require some web server scripting. These will typically interact subsequently via command scripts with the specific API of the FIRE resources, possibly using some predefined reservation and/or experimentation descriptions such as Resource Specification (RSpec) and OMF. The adapters are thus responsible for translating widget requests to FIRE APIs and vice versa. This way, the web client of the student does not address the FIRE resource directly but the adapters are making the request on behalf of the student and are as such speaking for them.

As explained before, we also aim at offering a generic platform for sharing existing courses while integrating them seamlessly in an e-Book or LMS. Therefore a "Lab Course import" process can be followed when a teacher wants to import a shared published course from the repository to a local specific instantiation to be used for a course. The activity diagram presented in Fig. 4 depicts the process. 


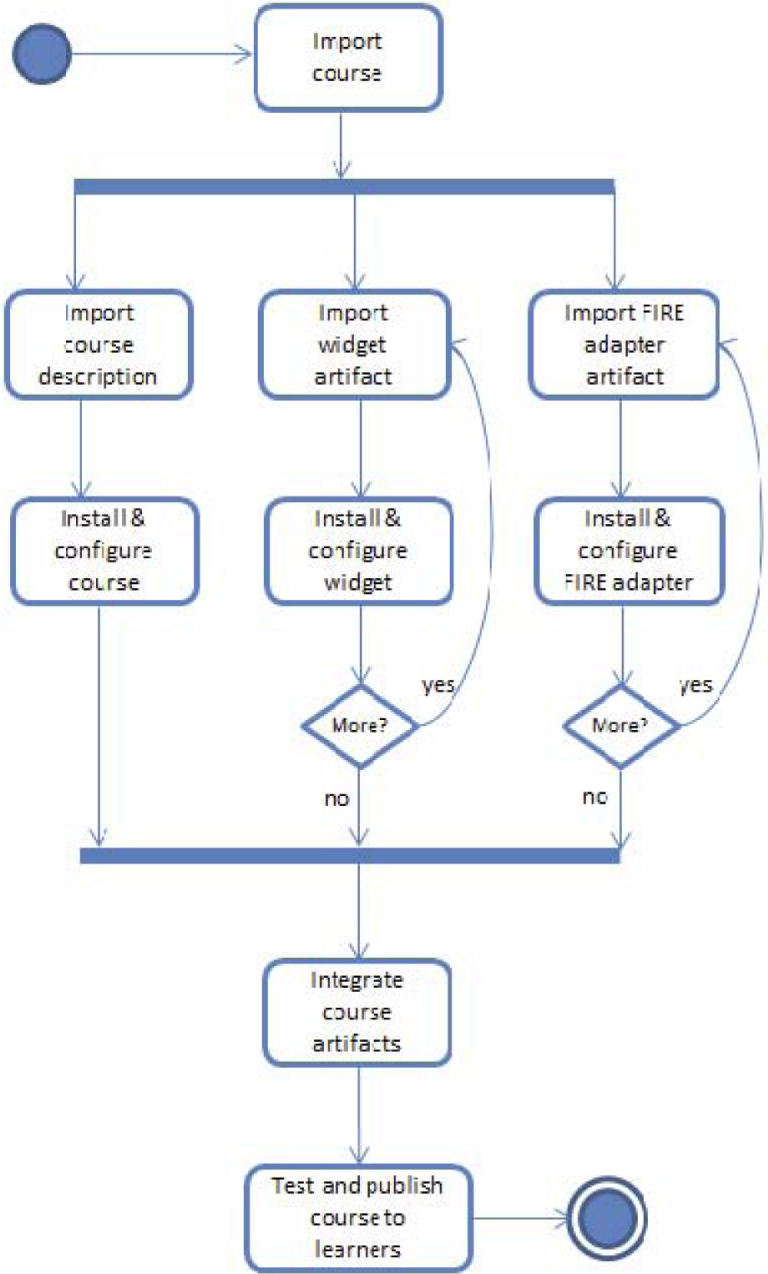

FIGURE 4. Importing existing labs in an iBook.

A first instantiation platform of our sharing repository is called FORGEStore and is deployed at http://www. forgestore.eu. FORGEStore is a marketplace for FORGEBox consumers, eBooks and LMSs. FORGEStore publishes widget end points, FORGEBox services, FIRE adapters and shared interactive courses packaged as SCORMs. FORGEStore is offered as open source and provides a REST API that can be easily consumed by third party web services. ${ }^{6}$

\section{B. AUTHENTICATION AND RESOURCES MANAGEMENT}

During the first connection, the student will be required to authenticate using one of the authentication methods selected by the instructor. At the moment, our architecture provides authentication based on Google ID, OpenID and the GENI-ID. The GENI-ID is able to access any identities from the InCommon Federation system based on the Shibboleth framework for more than one hundred facilities in the US and EU. This authentication process is required only once per session on the eBook or the LMS.

\footnotetext{
${ }^{6}$ More information is provided at https://github.com/ctranoris/forgestore
}

After the authentication phase, students can asynchronously access testbed resources thanks to a scheduler in the backend. This scheduler is able to analyse the resources needed in the current experiment based on a rich description and thus deploy the experiments as soon as resources are available. In this scenario, students can initiate all the experiments needed for the labs and they will be notified by email upon completion of these experiments.

In order to access and analyse the results, students use a specific widget where all their experiment results are accessible. In this widget, the student can analyse the measurements associated with any given experiment using the $\mathrm{R}$ statistical framework. Here, two options are available, either write $\mathrm{R}$ scripts or use predefined visualisation tools. These tools need to be prepared by the teacher. Once students finish their analysis, they can submit their answer using the same widget and the teacher will be able to access their answers using the web-based interface.

\section{EVALUATION METHODOLOGY}

Existing and commonly deployed quantitative and qualitative evaluation methods, such as questionnaires and surveys of educators and learners, have been adapted and augmented in order to meet specific requirements for evaluating the use of the FORGE courseware. For this purpose, we have developed two templates of questionnaires targeting two distinct groups of stakeholders: teachers and students. ${ }^{7}$

The template targeting teachers is focused on the quality and reusability of the offered learning resources. The questions are grouped under 3 main categories:

1) Quality of Content,

2) Potential Effectiveness,

3) Ease of Use.

The above categories include a number of statements about the FORGE learning materials where the responses are recorded as a 5-Likert scale ranging from 1 (strongly disagree) to 5 (strongly agree). These statement range from a very generic course appreciation such as "The teaching/ learning objectives of the FORGE learning materials can be easily identified." to more educational oriented ones such as "The FORGE learning materials include interactive instructional activities to improve teaching and learning." Additionally, open-ended questions have been included to record the teachers' views on possible improvements of the FORGE learning materials and additional comments.

The students' template is shorter and more focused on the ease of use and learning effectiveness of the learning materials and the learning activities that make use of FIRE facilities. The questionnaire is mainly comprised of a set of statements to which the student is requested to record his/her level of agreement via a 5-Likert scale ranging from 1 (strongly disagree) to 5 (strongly agree). Such statements

\footnotetext{
${ }^{7}$ These forms are available at https://emdesk.eu/shared/54ee2eeaa3dccb5d751d621234412b8b48a9af2c57fdb and https://emdesk.eu/shared/ 54ee2ec59dd7b-c09961558b15b32a114c6764fe 707208
} 
include for example "The content of this course was easy to understand." or "This course allowed me to control the rate, order and process of my learning." Similarly to the teachers' questionnaire, there are also some open-ended questions, asking about the things that the student has enjoyed most about the FORGE learning materials, possible improvements, as well as any additional comments.

Additionally, we are using quantitative evaluation methods based on Learning Analytics. Learning Analytics is the "measurement, collection, analysis and reporting of data about learners and their contexts, for purposes of understanding and optimizing learning and the environments in which it occurs" [30]. The field of Learning Analytics is essentially a "bricolage field, incorporating methods and techniques from a broad range of feeder fields: social network analysis (SNA), machine learning, statistics, intelligent tutors, learning sciences, and others" [31] . Learning Analytics applies techniques from information science, sociology, psychology, statistics, machine learning, and data mining to analyze data collected during education administration and services, teaching, and learning. Learning Analytics creates applications that directly influence educational practice [32].

To support monitoring and reporting on user activity, the FORGE architecture includes components that embrace the usage of Learning Analytics. The idea is to know when and which user experienced some content or interacted with a resource. Within FORGEBox we promote the usage of Experience API or xAPI or also known as the The Tin Can API: a specification for learning technology that makes it possible to collect data. There are many open source implementations around TinCan API for many well-known programming languages and therefore is our choice for FORGE to build on top of it Learning Analytics.

While learners experience a course, xAPI generates learning records. A Learning Record Store (LRS) is a data store system that serve as a repository for xAPI learning records. Therefore the presence of an LRS is mandatory in the FORGEBox reference architecture. Usually it is very easy for developers to use an LRS, since it is a simple RESTful endpoint which users that want to track their learners have an account. We present in Fig. 5 how we are facilitating the deployment of learning analytics in FORGE learning materials.

\section{COURSE CONTENT}

In this section, we present a representative subset of courses already available in FORGE. As detailed previously, associated course and labs descriptions are available on the FORGEStore website.

\section{1) ACM CS CURRICULUM COMPLIANCE}

a: EVOLUTION OF HTTP AND RELIABLE PROTOCOLS

As emphasised in the CS Curriculum, application layer and mechanisms to provide reliable communication are at the cornerstone of the Network and Communication component.

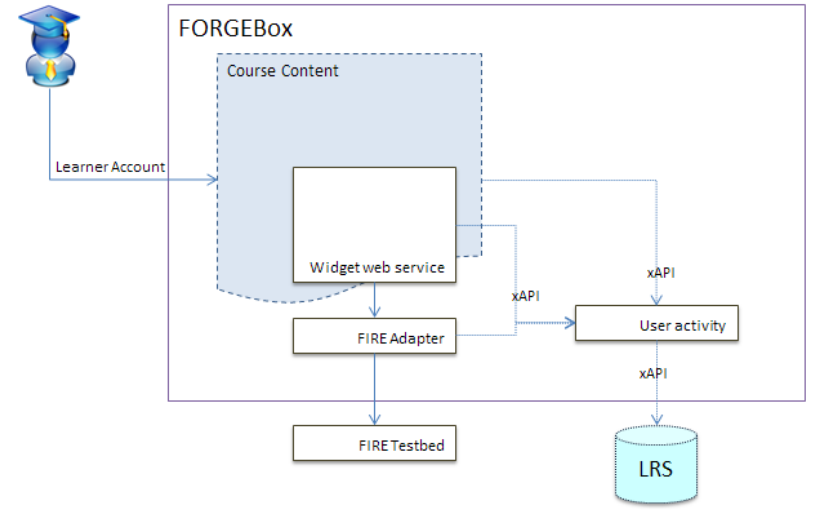

FIGURE 5. Enabling Learning Analytics in FORGE entities.

We have developed two widgets allowing us to illustrate the learning outcomes associated with both of these modules. The first widget allows us to build a lab demonstrating the evolution of the HTTP protocol from the original version 1.0 to version 1.1 and analysing the drawbacks of each version. In the second widget, the instructor can build a lab guiding the students through the development of various mechanisms to provide reliable data transfer on top of an unreliable channel. These labs illustrate the importance of pipelining mechanisms, and help the students to understand how to implement such mechanisms. We illustrate in Section IV how these widgets were used in our telecommunications course.

The two widgets provide several key services enumerated in Section III-A. First, they allow the teacher to reserve and provision necessary resources from the GENI and FIRE pool using a web-based interface. Second, these widgets interface within the GENI-ID authentication services to provide transparent identification to students from more than one hundred institutions in the US and EU. Third, they allow asynchronous access to resources thanks to an advanced scheduler based on the resources needed in the current experiment. Last, they provide the student with a analytical toolbox based on the $\mathrm{R}$ language.

\section{b: WIRELESS COMMUNICATION}

We have developed a set of widgets and adapters which enables learners to explore the characteristics of Wireless Local Area Network (WLAN) communication between wireless stations. The widgets control live experiments which employ wireless nodes in a large scale testbed for different scenarios of Wi-Fi communication. These scenarios involve one or multiple clients and various parameters such as packet size, QoS class, or physical bitrate. Results of live experiments are then displayed to the learner, who can interpret these results according to their understanding of the $\mathrm{Wi}-\mathrm{Fi}$ protocols.

The WLAN widget bundle consists of three separate widgets for controlling, monitoring and visualizing a FIRE experiment. They allow seamless integration on the platform in which they are used (e.g. a responsive course website, an e-book or an LMS). For this reason, the presentation of 
the widgets is based on the Bootstrap framework, giving a flexible HTML5 responsive basis for visualization in many different environments.

\section{c: TCP CONGESTION}

The TCP congestion control course provides a complete set of learning material, tools and infrastructure for conducting a series of experiments using both TCP and UDP. Through this interactive material, learners have the ability to run and, on-the-fly, manage experiments in a remote testbed with reserved hosts and network resources. In this environment, students have to perform experiments that examine various aspects of TCP congestion control. In particular, they can run and manage each test using a web-based interface that gives them remote access to the end nodes that run the servers and the clients. Furthermore, through this interface, users are able to connect to the experiment nodes, change all the necessary parameters for each test (e.g. delay, bandwidth), and also collect the results of a test to create the required output report.

This lab is based on a widget called "ssh2web", a web service providing three types of services. First, it offers a web-based ssh access to FIRE or GENI consortium nodes. Underlying this service, the widget manages essential services such as public key deployment and account creation and access control to testbed machines. Second, it offers visualization of the measurement stream. Last, it gives access to experiments' logs files. Overall, this widget covers the Resource Allocation and some parts of the Reliable Data Delivery (TCP part) learning outcomes from the ACM Curriculum.

\section{d: CAPACITY PLANNING OF COMPUTER SYSTEMS}

In this course, students learn techniques for performance evaluation of distributed systems. These techniques are then applied to design systems with good performance, and to the analysis of future workloads and the system changes required to cope with them. In this regard, our lab highlights the use of a traffic generating application and a network emulator to perform network analysis. The traffic generating application we are using is the Distributed Internet Traffic Generator (D-ITG) [33], an application capable of generating traffic that reproduces packet inter-arrival times and packet sizes of many common distributions, (e.g. exponential, normal, Pareto). The students perform a $3 \times 3$ factorial performance evaluation varying the value of the queue length in the network as well as the aforementioned inter-packet time distributions. Overall students have to configure around 80 experiments each on our system and an experiment takes around 4 minutes. Overall, this widget covers the Resource Allocation learning outcomes from the ACM Curriculum.

\section{2) CISCO ACADEMY COURSE}

The Cisco Academy course introduces learners to the basics of network equipment and configuration, using the Packet Tracer (PT) network simulator from the Cisco Systems Networking Academy. Within this course, PT is used by learners in order to reproduce different types of networks and understand how network technologies work and interact with each other. Furthermore, PT simulation results can be used in real-life Cisco network equipment.

The purpose of this course is to teach the basics of networks and the use of Cisco network equipment. In particular, learners who take this course should acquire an understanding of: the basics of IP addressing; the role of a router and a switch; server technology; how to configure IP addressing on a personal computer; how to send traffic from one device to another across a network; how to configure a router, with an address and a hostname; how to use router show commands to check its status; how to configure a router via its commandline interface.

We have developed a widget to embed a live connection to a running instance of PT inside an iBook or LMS. This enables learners to experiment with simulations of various types of networks without leaving the iBook or the LMS. These simulations are presented to learners in the context of interactive exercises, thus offering reflection and selfassessment opportunities throughout the course. The course also offers a variety of instructional videos demonstrating the use of network equipment. Fig. 6 shows a screenshot from the iBook version of the course, featuring an introduction to network routers and a video demonstrating a Cisco router.

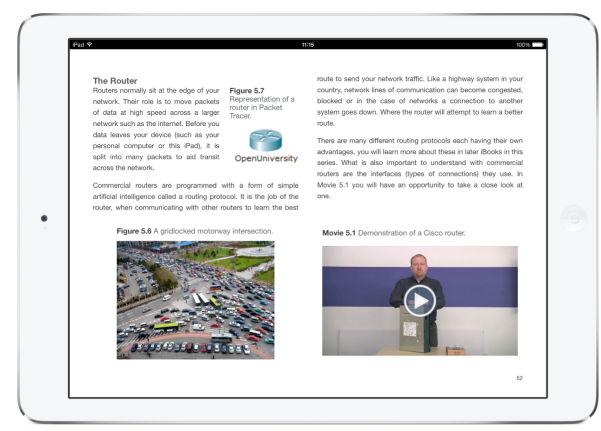

FIGURE 6. Extract from the iBook version of the Cisco Academy course.

In terms of implementation, rather than developing a full Packet Tracer Multi-user Protocol (PTMP) client in JavaScript, we made use of the existing Java PTMP libraries provided alongside PT. These libraries implement a set of classes corresponding to the main concepts in PT: Network, Device, Link, and so on, allowing a Java application to communicate with a running instance of PT, query its current status (current displayed network configuration, etc.), make changes, start and stop simulations and query simulated network behaviour. Since we cannot run any Java applications within the iBook. We therefore provide a way for the iBook widget to communicate with the Java application remotely. For further implementation details, we refer the reader to [34].

This course is not tied to any particular testbed, but aims to offer a bridge between the testbed community and the Cisco Networking Academy. The Cisco Networking 
Academy delivers a curriculum to help students develop the foundational ICT skills needed to design, build and manage networks. In order to deliver this curriculum, Cisco partners with educational institutions, non-profits, nongovernmental organisations, as well as community centres worldwide that provide classroom space, computer lab equipment, and qualified instructors. Cisco provides online curricula, virtual learning tools, instructional support, teacher training, and professional development opportunities for instructors.

We aim at exposing the millions of students of the Networking Academy to the educational services offered by the FIRE testbeds. Students who complete the Cisco course will be offered the option of taking other FORGE prototype courses, which feature exercises running on FIRE testbeds. In this way, they will be able to make the transition from experimenting with simulated networks to using real-life ones and apply what they have learned from PT to FIRE testbeds.

\section{DEPLOYMENTS}

\section{A. WIRELESS COMMUNICATION}

A preliminary lab course, using the iMinds w-iLab.t large scale wireless FIRE testbed, was first deployed in Ghent University, Belgium in October 2011. This lab course was repeated in 2012 and 2013 and execution on the FIRE resources was gradually further automated and refined, but still involved a lot of manual tweaking and preparation. In early 2014 we were able to successfully reuse the same lab course for teaching wireless communication to students at the premises of Trinity College Dublin (TCD), Ireland, while still using the hardware of the w-iLab.t facility in Belgium. This strengthened our belief in the potential of teaching lab courses anywhere worldwide with the use of state-of-the-art equipment which does not have to be situated nor maintained in-house but which is available on a FIRE facility. By complying to the FORGE architecture and using a set of widgets and adapters (see III-D), we were able to remove all manual interventions for preparing a lab session. A teacher now only needs to book a time slot online and students can perform wireless experiments via a sleek web interface. ${ }^{8}$

At Ghent University, this improved lab session was taught in February 2015 to 97 students. The lab session was organized to last for 2-3 hours and to take place in a computer lab room where students can perform the lab exercises with coaching of two university staff members to answer student questions. The different exercises (with FIRE experiments) are accompanied with one or multiple questions at the web interface. These questions are forcing students to think things over and to gain a better insight in wireless communication theory. Students expressed a great appreciation for this improved lab as shown by the fact that $92 \%$ of the students rated the lab with a score of 4 or 5 on a 5 -level Likert scale. Following the successful execution at Ghent University, this lab was very easily successfully reused at TCD, in March 2015 for about 25 students and at

\footnotetext{
${ }^{8}$ http://forge.test.iminds.be/wlan/
}

Universidade de Brasil (UnB), Brasil, in May 2015 for about 20 students. Other universities have also expressed their interest in reusing the lab.

Furthermore, Learning Analytics have been integrated into the lab to anonymously log the interactions of the students with the web interface into a Learning Record Store (LRS), using the open source Learning Locker for data storage and xAPI as the interface between the website and the LRS. This data will serve to improve the lab based on common student behaviour or mistakes.

\section{B. TCP CONGESTION LAB}

A first trial of the SSH2WEB widget and the TCP Congestion lab, described above, was performed in an undergraduate course in spring 2014 with more than 200 students at the University of Patras (UoP), Greece. In this course, we deployed and provisioned 150 nodes in a remote facility. The service was then used simultaneously by 50 users connecting via 3 instances of the widget, for a total of around 150 simultaneous connections. We repeated the scenario 4 times to accommodate the large number of students, and thus we had a chance to fix various issues. For example we managed to find issues relating to many pool connections on the web application's database, or abnormal usage by typing wrong credentials and URLs. During this prototype course, we also created some internal mechanisms that auto-registered students according to their University ID, to proper machines on the testbed.

Overall, the TCP Congestion Control Course used two separate labs, in order to evaluate the benefit of the FORGE toolkit approach. In the first lab, the students had to build an experiment using the testbed's native interface. In the second lab, this task was performed only using the previously introduced widgets. Students were asked to fill out a questionnaire after the labs. We present in Table 1 the results of this questionnaire. In this table, we show that in terms of difficulty the students rate the widget interface as significantly better. This preference is also reflected in the rating of the teaching and learning outcomes. Furthermore, we noticed a high demand from many students to perform the experiments again on their own as homework.

TABLE 1. Widget and native interface student rating (likert scale, 1:very hard - 5:easy, number of students).

\begin{tabular}{lccccc}
\hline \multicolumn{7}{c}{1} & 2 & 3 & 4 & 5 \\
\hline Native Interface \\
\hline Difficulty & 19 & 42 & 63 & 62 & 16 \\
Learning Outcome & 11 & 10 & 37 & 80 & 64 \\
\hline \multicolumn{7}{c}{ Widget Interface } \\
\hline Difficulty & 10 & 22 & 66 & 72 & 32 \\
Learning Outcome & 7 & 8 & 30 & 72 & 85 \\
\hline
\end{tabular}

UoP had setup the learning analytics tool called Learning Locker. Figure 7 depicts a sample collection of Learning Analytics statements from the lab course deployed 


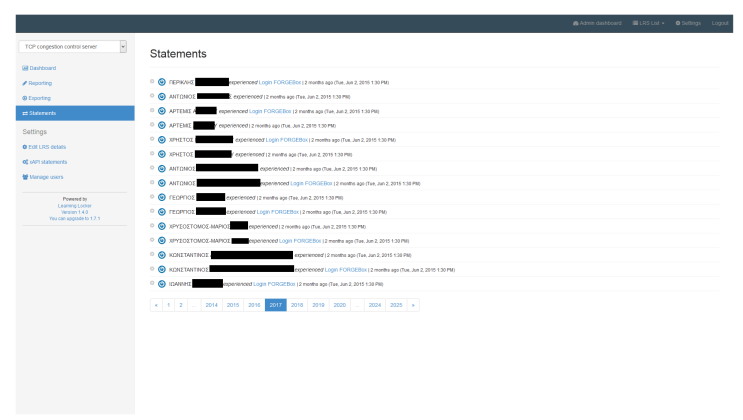

FIGURE 7. Learning analytics statements.

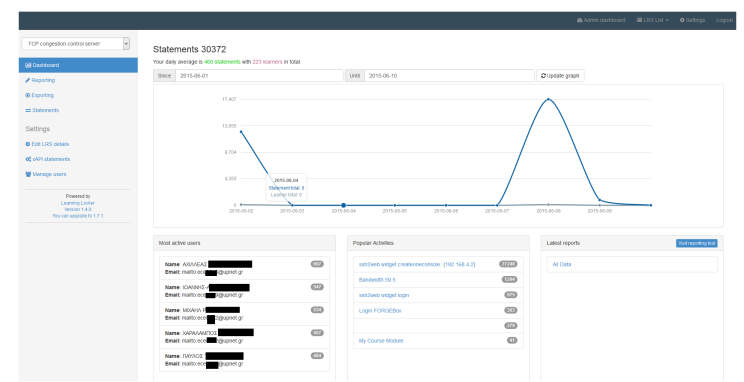

FIGURE 8. Students and popular activities records.

at June 2015 and Figure 8 displays the most active students and the most popular activities. The student organizational accounts (blurred in the figures) were used, thus we had the opportunity to identify and study all the actions that each student performed during the course within the Moodle LMS integrated with FORGE content and widgets, since our technologies are using the LTI standard. Some of the actions recorded for each student during the lab course were the following:

- Timestamp of page load of each section; That allows us to study the time needed to perform the whole activity but also to identify fast users against slow students and the mean average time of performing the activity.

- Score (correct answers) of the TCP congestion control questionnaire; Also the timestamp allows as to study the time to answer the questionnaire.

- Credentials used for login in widgets.

- Actions (commands, scripts, programs etc.) executed in a ssh2web widget; This is used to study what the students typed, when and if they did any other actions (e.g. the explored further the activity)

- Results taken after the experiment.

\section{CAPACITY PLANNING}

During the spring session 2014, our platform was used in its web-based variant in a post-graduate course at the UNSW, in Australia. Overall, 87 students between 22 to 35 years old were enrolled in the class. Out of this total number of students, a large subset (71) performed at least one experiment, mainly because they could chose to do this lab or a more mathematically based assignment. The students were given 2 months to conduct all their experiments and analyse the results.

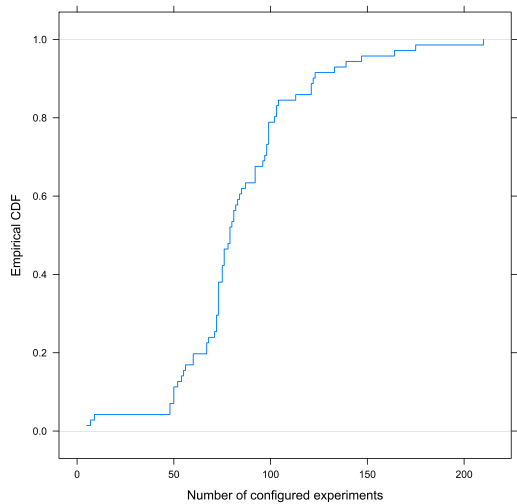

FIGURE 9. Number of experiments per user.

Nearly 6000 experiments were executed in a six-week span. We present in Fig. 9 the cumulative distribution function of the number of experiments performed per user. In this figure we can see that the median number of experiments is what we expected to be required to successfully answer the questions of the lab. Furthermore, we can see in Fig. 9 that three students did not configure enough experiments to answer the question and we found out that they did not submit anything.

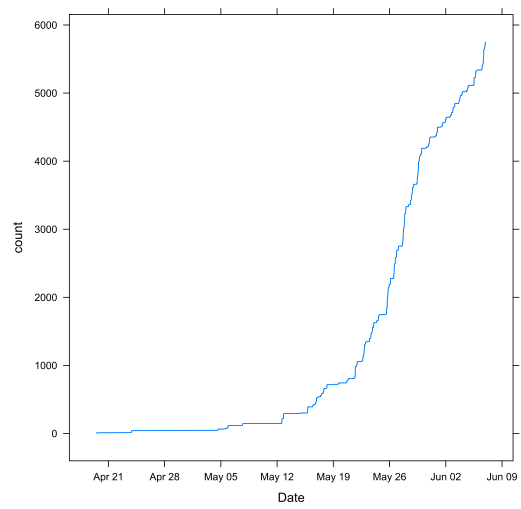

FIGURE 10. Time of creation of the experiments.

In Fig. 10, we represent the temporal cumulative number of experiments created by the students. The platform was opened at the beginning of April but no experiments were run for nearly three weeks. The deadline for the submission of the assessment was on June 7, and we can see on Fig. 10 that $85 \%$ of the experiments were performed at least before the weekend preceding the deadline. Finally, we have corroborated that once the deadline for the assignment was due, activity on the platform ceased entirely.

In Figure 11, we show the impact of this concentration of experiments, between May 22 and June 2, on the waiting time experienced by the students to retrieve their results. 


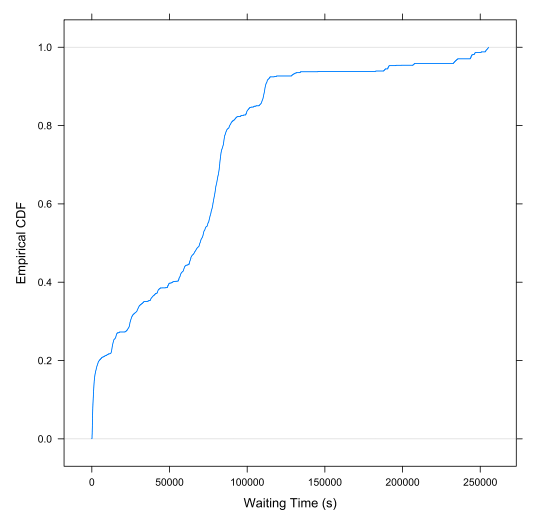

FIGURE 11. Waiting time before the experiment finished, in seconds.

As explained above, an experiment would take around 4 minutes to run, and our testbed was limited to running two experiments in parallel. Therefore we can see in Fig. 11 that the median waiting to retrieve the results was around 1 day due to the burst in configured experiments as shown in Fig. 10. We can also see that only a few experiments experienced long delays, which can be correlated with an IT outage on a Thursday which was not resolved until the Monday morning.

\section{USE-CASE COURSE}

We also adopted the framework presented in this paper in Trinity College Dublin, in Ireland to provide engineering students with enhanced hands-on laboratory experiences during the spring semester 2014. In particular, we offered online labs and remote experimentation to illustrate electronic engineering topics related to wireless communications, combined with lecturing for the wireless networks and communications module of a Master in Engineering. This allowed us to attest to the benefits of blended teaching, combining traditional lectures with hands-on experimentation using remote high-performance facilities, and its impact on the students' learning experience. Moreover, this approach enabled SRL, allowing the students to learn at their own pace. SRL describes the ability of students to learn how to learn. From a cognitive perspective, it enables students to become "motivationally, metacognitively and behaviourally active participants in their own learning process" [35].

Students were introduced to practical hands-on knowledge of telecommunications networks. The theory was complemented with practical work. Students were able to experiment with wireless communications and networks using three different high-performance testbeds located world-wide. Overall students performed more than 700 experiments, trying different configurations, and learnt from the system's feedback. The feedback from the students was very positive. They have expressed that they were able to get new skills on hands-on networking and also during the semester they have highlighted the most useful and practical content that they were able to apply on their studies.

\section{E. PROFESSIONAL WORKSHOPS}

The FORGE toolkit described in Section III was recently used at the first Fed4FIRE-GENI Research Experiment Summit. During that event, about 20 students, and researchers from universities and industry participated in Labwiki based hands-on tutorials on how develop and orchestrate experiments using resources from both GENI and FIRE testbed facilities. They went through a series of incremental exercises asking them to develop simple experiments using the Labwiki interface. The response to these tutorials was very positive, with many participants completing most of the assigned tasks and providing valuable feedback on existing features and potential improvements.

Early this year, the Labwiki widget was also used in tutorials at two consecutive GENI Engineering Conferences $\left(19^{\text {th }}\right.$ and $20^{\text {th }}$ GECs) as a platform to develop and execute experiments with resources distributed across several GENI-partner US universities. These tutorials were attended by 20 to 30 participants. The majority of the feedback from the last tutorial (about 75\%) positively rated it as helping participants to "gain general familiarity with the technology", and "get started using the capability hands on". These feedback, while impressive, have to be examined with a more critical mind as it might be possible that the nature of the audience had a significant impact on them. Indeed, in both the summer school and the GENI Engineering Conferences, the audience was highly motivated to use the presented tool as it would offer them a new channel for their own promotion of GENI and FIRE facilities.

\section{CONCLUSIONS}

We have presented a new approach for the deployment of potentially large scale distributed labs in well established eLearning platforms such as e-books and LMS. This approach allows educators to leverage these existing tools and user interface while seamlessly integrating resources from a large pool of federated facilities used in research to solve the institutional challenges mentioned in the ACM/IEEE CS 2013 curricula concerning the need for more heterogeneous hardware in labs. Furthermore, we have integrated state-of-the art authentication mechanisms allowing any students from more than one hundred US and EU Universities to already access these resources.

Our solutions also allows teachers to lower the accessibility threshold for students to access these resources, thanks to an integrated automatic generation of widgets for eBooks, thus offering a familiar interface to students.

We demonstrated, through on a set of widgets, that we can already cover most of the requirements from the Network and Communication component of the CS 2013 curriculum and some of the labs of the Cisco academy. We presented experience report illustrating the benefits of this framework based on deployments in four university courses and in professional workshops. In particular, we demonstrated the robustness of our platform based on its deployment during a post-graduate 
course during the spring 2014 semester. In this class more than 6000 experiments were provisioned, configured and executed in the span of a month and half.

\section{REFERENCES}

[1] "ACM/IEEE-CS joint task force on computing curricula, computer science curricula 2013," ACM/IEEE Computing Curricula, Tech. Rep., Dec. 2013. [Online]. Available: http:http://www.acm.org/education/ CS2013finalreport.pdf

[2] M. Berman et al., "GENI: A federated testbed for innovative network experiments," Comput. Netw., vol. 61, pp. 5-23, Mar. 2014.

[3] A. Gavras, A. Karila, S. Fdida, M. May, and M. Potts, "Future Internet research and experimentation: The FIRE initiative," ACM SIGCOMM Comput. Commun. Rev., vol. 37, no. 3, pp. 89-92, Jul. 2007. [Online]. Available: http://doi.acm.org/10.1145/1273445.1273460

[4] GENI Education. [Online]. Available: http://www.cs.unc.edu/ Research/geni/geniEdu/, accessed Jan. 6, 2016

[5] Seattle, Open Peer-to-Peer Computing. [Online]. Available: https://seattle.cs.washington.edu/html/, accessed Jan. 6, 2016.

[6] N. Handigol, B. Heller, V. Jeyakumar, B. Lantz, and N. McKeown, "Reproducible network experiments using container-based emulation," in Proc. CoNEXT, 2012, pp. 253-264.

[7] G. Jourjon, S. Kanhere, and J. Yao, "Impact of an e-learning platform on CSE lectures," in Proc. ITiCSE, 2011, pp. 83-87.

[8] G. Jourjon, T. Rakotoarivelo, and M. Ott, "From learning to researching, ease the shift through testbeds," in Proc. Tridentcom, 2010, pp. 496-505.

[9] Y. Zhuang et al., "Taking a walk on the wild side: Teaching cloud computing on distributed research testbeds," in Proc. SIGCSE, 2014, pp. 535-540. [Online]. Available: http://doi.acm.org/10.1145/2538862.2538931

[10] T. de Jong, "Scaffolds for computer simulation based scientific discovery learning," in Dealing With Complexity in Learning Environments, J. Elen and R. E. Clark, Eds. Amsterdam, The Netherlands: Elsevier, 2006, pp. 107-128.

[11] K. Hakkarainen, "Emergence of progressive-inquiry culture in computersupported collaborative learning," Learn. Environ. Res., vol. 6, no. 2, pp. 199-220, May 2003.

[12] E. Kasl and L. Yorks, "Collaborative inquiry for adult learning," in New Directions for Adult and Continuing Education, vol. 94. Hoboken, NJ, USA: Wiley, 2002.

[13] P. Mulholland et al., "nQuire: Technological support for personal inquiry learning," IEEE Trans. Learn. Technol., vol. 5, no. 2, pp. 157-169, Apr./Jun. 2012.

[14] K. Steffens, "Self-regulated learning in technology-enhanced learning environments: Lessons of a European peer review," Eur. J. Edu., vol. 41 nos. 3-4, pp. 353-379, Sep./Dec. 2006

[15] T. Berners-Lee. (2006). Linked Data-Design Issues. [Online]. Available: http://www.w3.org/DesignIssues/LinkedData.html

[16] C. Bizer, T. Heath, and T. Berners-Lee, "Linked data-The story so far," Int. J. Semantic Web Inf. Syst., vol. 5, no. 3, pp. 1-22, 2009

[17] T. Berners-Lee, J. Hendler, and O. Lassila, "The semantic Web," Sci. Amer., vol. 284, no. 5, pp. 28-37, 2001

[18] S. McIntyre and N. Mirriahi. (Aug. 2015). Learning to Teach Online. [Online]. Available: http://online.cofa.unsw.edu.au/

[19] A. Diwakar, S. Poojary, R. Rokade, S. Noronha, and K. Moudgalya, "Control systems virtual labs: Pedagogical and technological perspectives," in Proc. IEEE Int. Conf. Control Appl. (CCA), Aug. 2013, pp. 483-488. [Online]. Available: http://dx.doi.org/10.1109/cca.2013.6662796

[20] T. Rakotoarivelo, G. Jourjon, and M. Ott, "Designing and orchestrating reproducible experiments on federated networking testbeds," Comput. Netw., vol. 63, pp. 173-187, Apr. 2014.

[21] O. Mehani, G. Jourjon, T. Rakotoarivelo, and M. Ott, "An instrumentation framework for the critical task of measurement collection in the future Internet," Comput. Netw., vol. 63, pp. 68-83, Apr. 2014.

[22] T. Rakotoarivelo, M. Ott, G. Jourjon, and I. Seskar, "OMF: A control and management framework for networking testbeds," ACM SIGOPS Oper. Syst. Rev., vol. 43, no. 4, pp. 54-59, Jan. 2010. [Online]. Available: http://doi.acm.org/10.1145/1713254.1713267

[23] Y.-H. Kim et al., "Enabling iterative development and reproducible evaluation of network protocols," Comput. Netw., vol. 63, pp. 238-250, Apr. 2014.

[24] M. Pizzonia and M. Rimondini, "Netkit: Easy emulation of complex networks on inexpensive hardware," in Proc. 4th Int. Conf. Testbeds Res. Infrastruct. Develop. Netw. Commun. (Tridentcom), 2008, pp. 1-7.

[25] H. Nguyen, M. Roughan, S. Knight, N. Falkner, O. Maennel, and R. Bush, "How to build complex, large-scale emulated networks," in Proc. Tridentcom, 2010, pp. 3-18.

[26] PlanetLab Consortium. PlanetLab: An Open Platform for Developing, Deploying, and Accessing Planetary-Scale Services. [Online]. Available: http://www.planet-lab.org/, accessed Jan. 6, 2016.

[27] A. Wright, "Tablets over textbooks?" Commun. ACM, vol. 55, no. 3, pp. 96-104, Mar. 2012.

[28] J. B. Fenwick, B. L. Kurtz, P. Meznar, R. Phillips, and A. Weidner, "Developing a highly interactive ebook for CS instruction," in Proc. SIGCSE, 2013, pp. 135-140. [Online]. Available: http://doi.acm.org/10.1145/2445196.2445241

[29] M. Langer, iBooks Author: Publishing Your First eBook. Lea County, NM, USA: Flying M Production Inc., 2012.

[30] G. Conole and D. Gašević, Proc. 1st Int. Conf. Learn. Anal. Knowl. (LAK), 2011

[31] G. Siemens, "Supporting and promoting learning analytics research," J. Learn. Anal., vol. 1, no. 1, pp. 1-2, 2014

[32] S. B. Shum, "Learning analytics," UNESCO, Paris, France, Tech. Rep. 8378, 2012

[33] A. Botta, A. Dainotti, and A. Pescapè, "A tool for the generation of realistic network workload for emerging networking scenarios," Comput. Netw., vol. 56, no. 15, pp. 3531-3547, Oct. 2012

[34] A. Mikroyannidis, J. Domingue, A. Third, A. Smith, and N. Guarda, "Online learning and experimentation via interactive learning resources," in Proc. 3rd Experim. Int. Conf., 2015, pp. 1-6.

[35] J. M. Marquez-Barja, G. Jourjon, A. Mikroyannidis, C. Tranoris, J. Domingue, and L. A. DaSilva, "FORGE: Enhancing eLearning and research in ICT through remote experimentation," in Proc. IEEE Global Eng. Edu. Conf. (EDUCON), Apr. 2014, pp. 1157-1163.

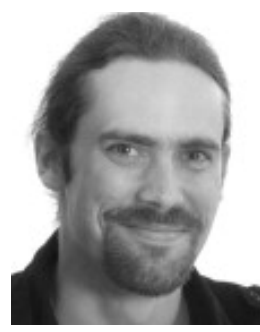

GUILLAUME JOURJON received the Engineering degree from ENSICA, the D.E.U.G. degree in physics and chemistry with a minor in mathematics from the University of Toulouse III, and the Ph.D. degree from the University of New South Wales and the Toulouse University of Science, in 2008. He is currently a Senior Researcher with Data61. His research areas of interest are related to the development of new teaching and learning facilities, measurement architecture and testbeds, and software defined network and network economics.

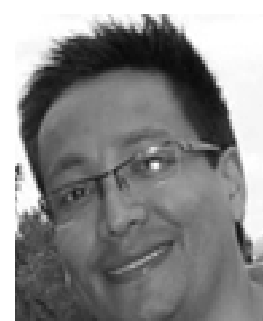

JOHANN M. MARQUEZ-BARJA received the Systems Engineering (Hons.) degree, the M.Sc. degree in telematics, the M.Sc. degree in computer architecture, and the Ph.D. (Hons.) (cum laude) degree in architecture and technology of computer systems from the Universitat Politecnica de Valencia, Spain. He is currently a Research Fellow with the CTVR Telecommunications Research Centre, Trinity College Dublin, Ireland. His main research areas of interest are wireless/small cells architectures and passive optical network backhauls

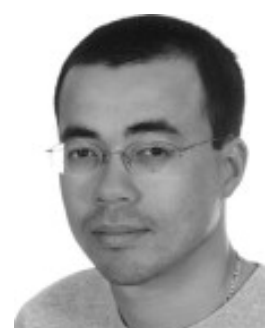

ments and analysis.
THIERRY RAKOTOARIVELO received the Ph.D. degree in a cotutelle agreement with UNSW and ENSICA, in 2007. He is currently a Senior Researcher with the Network Research Group, Data61, where he works on frameworks for large scale worldwide experimental studies. He is also interested in using these frameworks to improve the teaching of networking courses, using the IREEL eLearning Platform. He recently started new activities related to experimental measure- 


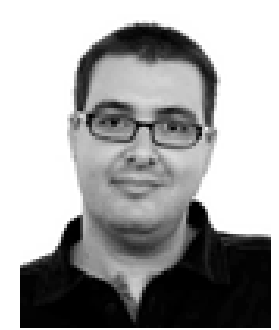

ALEXANDER MIKROYANNIDIS received the B.Eng. degree in electrical and computer engineering from the University of Patras, Greece, the M.Phil. degree in computation from the University of Manchester Institute of Science and Technology, and the Ph.D. degree in informatics from the Manchester Business School. He is currently a Research Associate with the Knowledge Media Institute, The Open University, U.K. His research areas of interest are related to knowledge management and applications of semantic and social Web technologies.

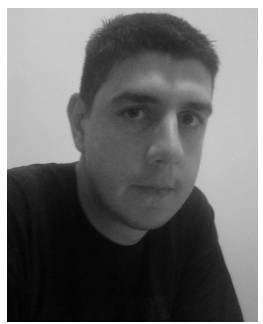

KOSTAS LAMPROPOULOS received the Diploma and Ph.D. degrees from the Electrical and Computer Engineering Department, University of Patras, Greece, in 2003 and 2012, respectively. He was a Firmware Engineer with the WLAN Group, Atmel Hellas, part of Multimedia \& Communications Group of Atmel Corporation (2003-2005). From 2010 to 2014, he was a member of the European Telecommunication Standards Institute. His research is focused on the areas of identity management, privacy, and security in next generation networks.

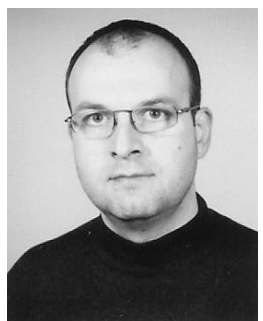

SPYROS DENAZIS received the B.Sc. degree in mathematics from the University of Ioannina, Greece, in 1987, and the Ph.D. degree in computer science from the University of Bradford, U.K., in 1993. Since then, he held numerous positions in several prestigious universities, research institutes, and the industry. He is currently an Associate Professor with the Electrical and Computer Engineering Department, University of Patras, Greece.

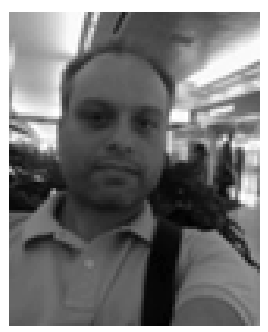

CHRISTOS TRANORIS received the Diploma degree from the Electrical and Computer Engineering Department, University of Patras, in the area of software processes on the modeling and design of industrial applications, in 1999, where he is currently pursuing the $\mathrm{Ph} . \mathrm{D}$. degree. Since 1995 , he has been on the private sector as a Software Engineer. He co-founded Instance Ltd., in 2001, a software company. After 2006, he was a Software Tech. Lead QE with the Bytemobile's European Development Center. He is currently a Senior Researcher with the Electrical and Computer Engineering Department, University of Patras.

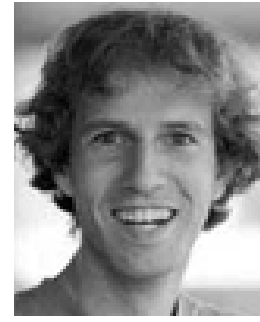

Research Institute.

DAAN PAREIT received the M.Sc. degree in electrotechnical engineering with a focus on ICT and the Ph.D. degree in computer science engineering from Ghent University, (UGent), Belgium, in 2006 and 2011, respectively. In 2006, he joined the Information Technology Department, UGent. Within this department, he is working with the Internet Based Communication Networks and Services research Group. As a member of this research group, he is also affiliated with the iMinds

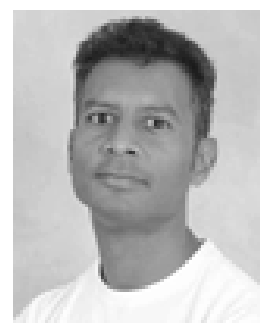

online learning.
JOHN DOMINGUE is currently the Deputy Director of the Knowledge Media Institute with The Open University. In the early 1990s, before moving to $\mathrm{KMi}$, he developed and later chaired the first OU course to be taught completely online and the first to handle assignments electronically writing both the course set book and the exams. Building upon his research in software visualization, the course set book was structured around an incrementally exposed virtual machine to facilitate

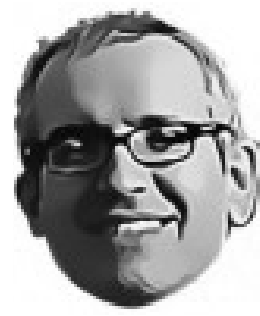

LUIZ A. DASILVA is currently the Professor of Telecommunications with Trinity College Dublin. He is also a Professor with the Bradley Department of Electrical and Computer Engineering, Virginia Tech, USA. His research focuses on distributed and adaptive resource management in wireless networks, and in particular wireless resource sharing, dynamic spectrum access, and the application of game theory to wireless networks. He is currently a Principal Investigator on research projects funded by the National Science Foundation in the USA, the Science Foundation Ireland, and the European Commission under Horizon 2020 and Framework Program 7. He is a Co-Principal Investigator of CONNECT, the Telecommunications Research Centre, Ireland.

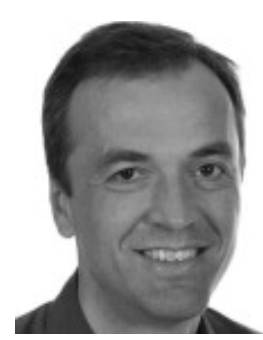

and Knowledge Management systems.
MAX OTT is a Senior Principal Researcher and Research Leader with the Networks Research Group, Data61. He is heavily involved in many of the worldwide experimental research facility activities, such as GENI (USA) and FIRE with many testbeds adopting the award-winning OMF control and management framework. Before coming to NICTA, he founded Semandex, which is a pioneer provider of content-based networks, a new generation of Enterprise Information Integration 